\title{
Modeling and Frequency Analysis of a Dual H-bridge Current Flow Controller in Meshed HVDC systems
}

\author{
Wei Liu, Jun Liang, C. E. Ugalde-Loo, and Gen Li \\ Cardiff University, Wales, UK \\ E-Mail: Liuw28@ cardiff.ac.uk \\ Chuanyue Li and Frederic Colas \\ L2EP, Lille, France \\ E-Mail: Chuanyue.Li@outlook.com
}

\section{Acknowledgements}

The authors gratefully acknowledge the funding from the project "Innovative Tools for Offshore Wind \& DC Grids (InnoDC)". This project has received funding from the European Union's Horizon 2020 Research and Innovation Program under the Marie Sklodowska-Curie grant agreement no. 765585.

\section{Keywords}

«Multi-terminal HVDC», «Current Flow Controller», «Dual H-bridge CFC», «Small-signal Modeling», «Frequency Analysis».

\begin{abstract}
This paper investigates the modeling and operation of dual H-bridge current flow controllers (CFCs) in meshed high-voltage direct-current (HVDC) systems. Two operating modes of the CFC device, namely the 'buck mode' and the 'boost mode', are defined and analyzed. Small-signal models of the dual Hbridge CFC are derived in the $s$-domain for each operating mode. A frequency sweep procedure is carried out using PSIM to obtain frequency domain representations of the CFC to verify the validity and accuracy of the small-signal models. The dynamic performance of the dual $\mathrm{H}$-bridge $\mathrm{CFC}$ is verified through time-domain simulations conducted in PSCAD/EMTDC.
\end{abstract}

\section{Introduction}

Voltage source converter (VSC) based HVDC systems are an attractive solution for the integration of large-scale renewable energy to ac grids [1]. The connection of existing point-to-point HVDC links with additional converter terminals to build multi-terminal dc (MTDC) grids has been proposed to enhance the feasibility and reliability of the whole system. However, there are still challenges to operate meshed MTDC systems safely [2], [3]. One of them is the current or power flow control within a dc grid [4]. The current flow between dc nodes in a meshed system is determined by the resistance of the transmission lines, which tends to be small. Line overloading may occur if the current flow is not regulated properly.

Different CFC topologies have been proposed in the literature to achieve current flow regulation in MTDC systems. A direct way to do this is to insert resistors into the dc transmission lines [5]. However, this method generates extra losses and large cooling systems are required to dissipate the excess heat. Another approach is to insert active dc sources to the transmission lines so that active power is absorbed or generated to change the current flow [6]. An active dc source exchanges power with an ac system, which can prevent large power losses. However, the adoption of this solution would require large isolation transformers which, in turn, would increase the capital cost and the footprint of the system.

An interline dual H-bridge CFC topology was presented in [7], [8] to reduce power losses and the capital cost. An advantage of this topology is that no isolation transformers are needed since the device 
exchanges power between dc transmission lines. In addition, this CFC configuration can be used to increase, decrease and even reverse the current flow of a transmission line in a meshed MTDC grid.

The basic operating principle and models of a dual H-bridge CFC have been investigated in [9]-[11]. An average model was used to analyze the dynamic performance of the CFC in meshed MTDC grids. However, the switching dynamics are estimated when average models are adopted. In addition, an understanding on how the device's representation is affected by changes in the operating mode is not clear. This represents an under-researched topic in the literature.

To bridge this research gap, the dual H-bridge CFC is investigated in detail in this paper. To provide insight, an equivalent circuit of the device is first obtained. Based on this simplified configuration, the operating modes of the CFC are classified as 'buck' and 'boost' modes. Small-signal models under these operating modes are derived in the $s$-domain. These representations explicitly show how the dynamic behavior of the device changes when the operating mode is modified. A frequency sweep is conducted in PSIM to obtain alternative representations of the device to verify the accuracy of the small-signal models. A good agreement is observed between the results obtained from small-signal models and from the frequency sweep, which provides credibility on the validity of the small-signal models. For completeness, the dynamic performance of the CFC is verified with time-domain simulations conducted in PSCAD.

\section{Principle of the dual H-bridge CFC}

\section{System configuration}

CFCs have been proposed to be used in meshed HVDC systems to regulate transmission line currents. To avoid unnecessary complexity and to provide a clear analysis, a three-terminal meshed MTDC system is adopted. The system configuration is illustrated in Fig. 1. The dual H-bridge CFC is implemented in Node 1 to regulate the current flow between lines a and $b$.

The basic topology of the device is shown in Fig. 2(a), which consists of two H-bridges with a common dc bus. As the two bridges connected with Node 1 are in parallel, a simplified topology, as shown in Fig. 2(b), can be obtained by removing switches $S_{1}^{\prime}$ and $\overline{S_{1}^{\prime}}$, The analysis of the dual H-bridge CFC will be based on this simplified topology [7].

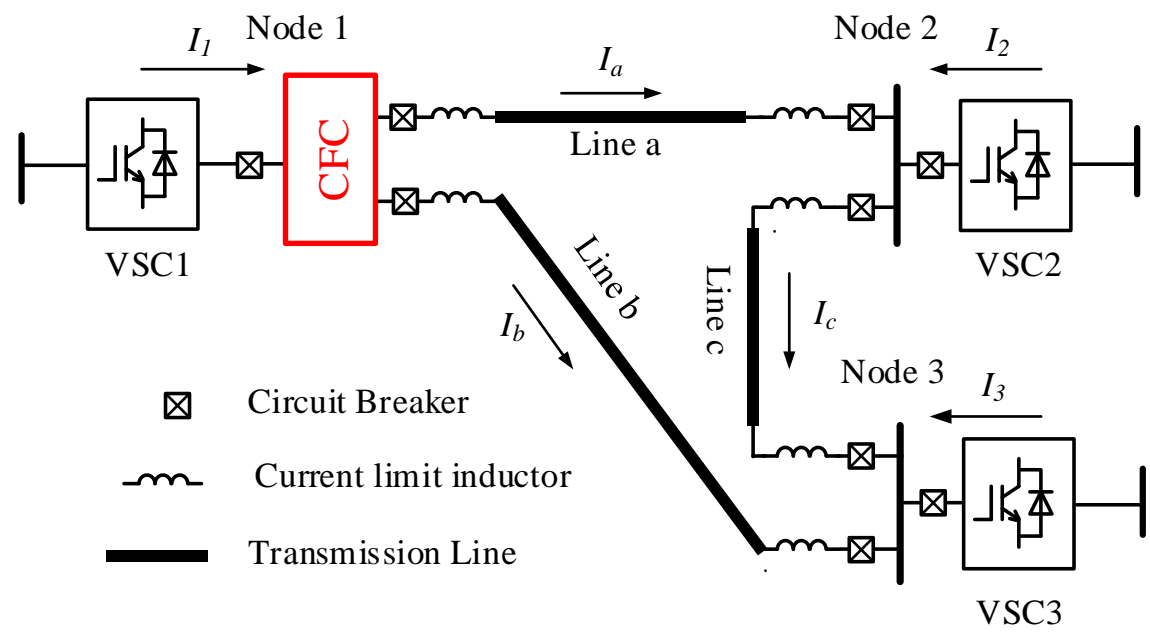

Fig. 1: Three-terminal HVDC system with the dual H-bridge CFC.

\section{Operating modes of the dual H-bridge CFC}

Two operating modes of the CFC, namely the 'buck mode' and the 'boost mode', are defined based on the relationship between the input current $I_{1}$ (the node current) and the output currents $I_{a}$ and $I_{b}$ (currents of the transmission lines). To analyze the basic operation principle of the device, ideal switches are used to represent the power electronic devices. This is shown in Fig. 3. If ' $S=1$ ', the switch is in an 'on' 
state. Conversely, ' $S=0$ ' implies that the switch is 'off'. If ' $S=\mathrm{PWM}$ ', the switch is regulated by a pulse-width modulation (PWM) signal. The switches in each bridge work complementarily.

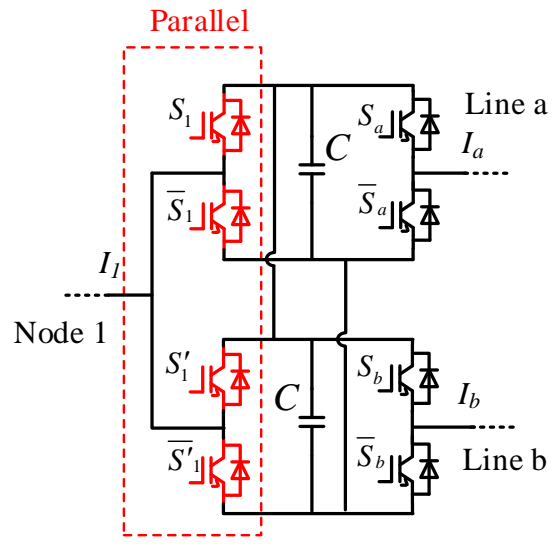

(a)

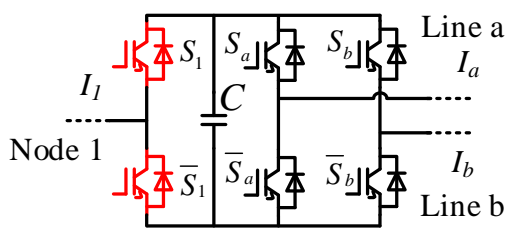

(b)

Fig. 2: (a) Schematic of the basic dual H-bridge CFC. (b) Simplified topology of the dual H-bridge CFC.

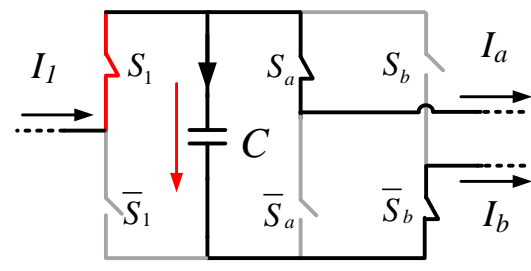

I: Charging

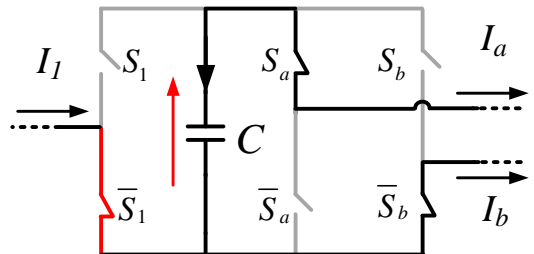

II: Discharging

(a) $S_{1}=P W M, S_{a}=1, S_{b}=0$

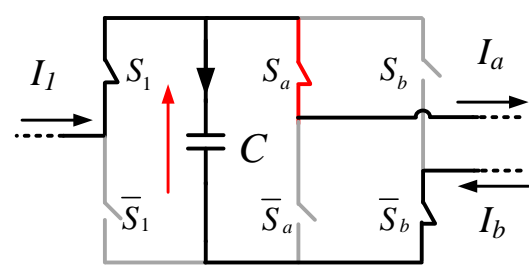

I: Dis charging

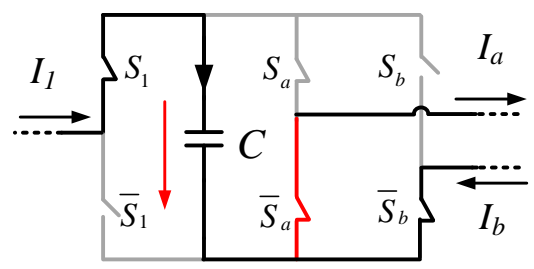

II: Charging

(b) $S_{1}=1, S_{a}=P W M, S_{b}=0$

Fig. 3: Operating modes of the CFC. (a) 'Buck mode'. (b) 'Boost mode'.

\section{'Buck Mode'}

The CFC works in 'buck mode' when a PWM signal is applied to switches $S_{1}$ and $\overline{S_{1}}$. At the same time, switch $S_{a}$ is kept in an 'on' state and switch $S_{b}$ is kept in an 'off' state, as shown in Fig.3 (a). Current $I_{1}$ is seen as constant since it is regulated by the VSC in the node. When $S_{1}$ is 'on', the current through the capacitor $i_{c}$ equals to $I_{1}-I_{a}$. Conversely, when $S_{1}$ is 'off', the current through the capacitor $i_{c}$ becomes $-I_{a}$. To ensure energy balance of the capacitor, the average current flowing through it should be zero in steady-state within one period of the PWM. The relationship of the current can be derived as:

$$
\begin{gathered}
\bar{I}_{c}=\frac{1}{T} \int_{0}^{T} i_{c}(t) d t=\frac{\left(I_{1}-I_{a}\right) \times D T-I_{a}(1-D) T}{T}=0 \\
I_{a}=I_{1} D, I_{b}=I_{1}(1-D)
\end{gathered}
$$

where $T$ is the period of the PWM and $D$ is the duty cycle. 
From equation (2), it can be seen that the currents in the transmission lines can be regulated using duty cycle $D$. As its value is always between 0 and 1 (i.e. $0 \leq D \leq 1$ ), the magnitude of line currents $I_{a}$ and $I_{b}$ will be less than or equal to the magnitude of node current $I_{1}$ under the 'buck mode'. Therefore, this operating mode can be used to balance the current flows between two transmission lines.

\section{'Boost Mode'}

The CFC works in a 'boost mode' when a PWM signal is applied to either switch $S_{a}$ or $S_{b}$. Taking $S_{a}$ as an example, this switch is regulated by PWM when $S_{1}$ is kept in an 'on' state and $S_{b}$ is kept 'off' (see Fig. 3(b)). When $S_{a}$ is 'on', the current through the capacitor $i_{c}$ equals to $I_{1}-I_{a}$, whereas when $S_{a}$ is 'off', the current through the capacitor becomes $i_{c}=I_{1}$. In steady-state, the average current flowing through the capacitor should be zero within one PWM period. This way, a relationship of the current can be derived as follows:

$$
\begin{gathered}
\bar{I}_{c}=\frac{1}{T} \int_{0}^{T} i_{c}(t) d t=\frac{\left(I_{1}-I_{a}\right) \times(1-\mathrm{D}) T+I_{1} D T}{T}=0 \\
I_{a}=\frac{I_{1}}{1-D}, I_{b}=\frac{-D I_{1}}{1-D}
\end{gathered}
$$

As in the 'buck mode', it can be seen from equation (4) that the currents of the transmission lines can be regulated with the duty cycle $D(0<D<1)$. However, under the 'boost mode' line current $I_{a}$ will be higher than node current $I_{1}$, while current $I_{b}$ will be reversed. The same result also applies to $I_{b}$ when the PWM signal is applied, instead, to $S_{b}$.

\section{Small-signal modeling of the dual H-bridge CFC}

The dual H-bridge CFC is a nonlinear time-varying device due to its switching process (PWM modulation). In order to analyze the dynamic behavior of the CFC and to design a suitable current controller, the switching process should be linearized. To focus on the analysis of the device, the transmission lines can be simplified as inductors and resistors and the VSCs represented by their average models (see Fig. 4). By using state-space averaging methods [12], the small-signal models of the CFC are derived for both the 'buck mode' and the 'boost mode'.

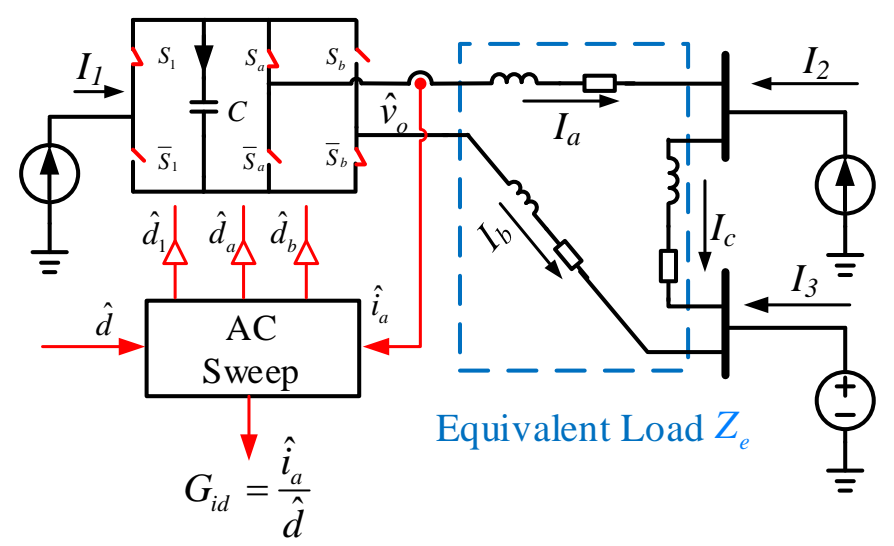

Fig. 4: Simplied HVDC system with the CFC. 


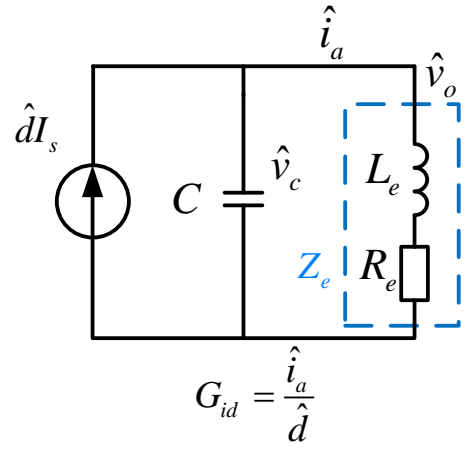

(a)

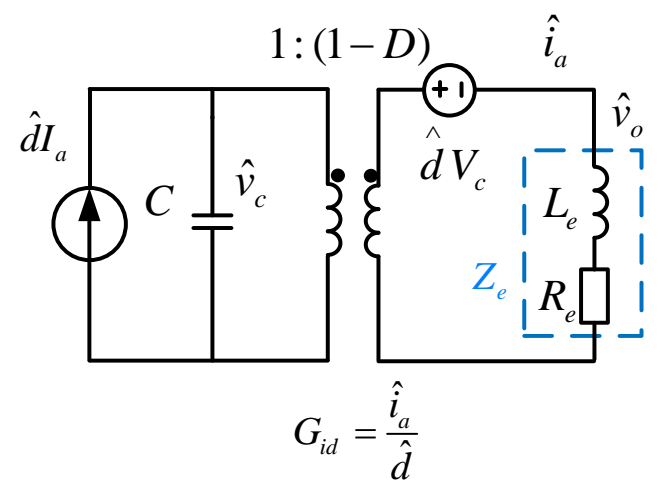

(b)

Fig. 5: Small-signal model of the CFC: (a) 'buck mode'; (b) 'boost mode'.

\section{'Buck mode' model}

For the 'buck mode', switching devices $S_{1}$ and $\overline{S_{1}}$ are regulated by a PWM signal. The small-signal model can be obtained by linearizing the CFC at a steady-state operating point. The differential equation for the capacitor and output voltage (seen by transmission lines) can be obtained as:

$$
\begin{aligned}
& \begin{cases}C \frac{d v_{c}(t)}{d t}=i_{s}(t)-i_{a}(t) & \text { from } 0 \text { to } d(t) \\
C \frac{d v_{c}(t)}{d t}=-i_{a}(t) & \text { from } d(t) \text { to } 1\end{cases} \\
& v_{o}(t)=v_{c}(t)=L_{e} \frac{d i_{a}}{d t}+R_{e} i_{a} \quad \text { from } 0 \text { to } 1
\end{aligned}
$$

where $i_{s}(t)$ is the node current, $i_{a}(t)$ is the current of line a, $v_{c}(t)$ is the capacitor voltage of the CFC, $v_{o}(t)$ is the output voltage, $d(t)$ is the PWM duty cycle.

An average switching model of the CFC can be derived based on equations (5) and (6) by calculating the average values of relevant variables within one duty cycle:

$$
C \frac{d\left\langle v_{c}(t)\right\rangle_{T}}{d t}=\left(i_{s}(t)-i_{a}(t)\right) d(t)+\left(-i_{a}(t)\right)(1-d(t))=i_{s}(t) d(t)-i_{a}(t)
$$

However, the switching model is still non-linear. To obtain a linear model, a small increment $\hat{x}$ is considered to the steady-state operating point $X$. In other words, a variable can be expressed as $x(t)=X+\hat{x}$. Substituting $v_{c}(t)=V_{c}+\hat{v}_{c}, i_{s}(t)=I_{s}+\hat{i}_{s}, i_{a}(t)=I_{a}+\hat{i}_{a}, d(t)=D+\hat{d}$ into (6) and (7) and by neglecting the second order and dc components, a linearized model can be obtained,

$$
\left\{\begin{array}{l}
C \frac{d \hat{v_{c}}}{d t}=\hat{i_{s}} D+\hat{d} I_{s}-\hat{i}_{a} \\
L \frac{d \hat{i_{a}}}{d t}+R \hat{i_{a}}=\hat{v_{c}}
\end{array}\right.
$$

Fig. 5(a) gives the equivalent circuit of the small-signal model of the CFC for the 'buck mode'. By applying the Laplace transform to equation (8), a transfer function from the line current $\hat{i}_{a}$ to the duty cycle $\hat{d}$ is derived as follows: 


$$
G_{i d}=\frac{\hat{i}_{a}}{\hat{d}}=\frac{I_{1}}{L_{e} C s^{2}+R_{e} C s+1}
$$

\section{'Boost mode' model}

For the 'boost mode', the differential equation for the capacitor and the output voltage of the CFC are be obtained as follows:

$$
\begin{aligned}
& \begin{cases}C \frac{d v_{c}(t)}{d t}=i_{s}(t)-i_{a}(t) & \text { from } 0 \text { to } d(t) \\
C \frac{d v_{c}(t)}{d t}=i_{s}(t) & \text { from } d(t) \text { to } 1\end{cases} \\
& \begin{cases}v_{o}(t)=v_{c}(t) & \text { from } 0 \text { to } d(t) \\
v_{o}(t)=0 & \text { from } d(t) \text { to } 1\end{cases}
\end{aligned}
$$

where $i_{s}(t)$ is the node current, $i_{a}(t)$ is the current of line a, $v_{c}(t)$ is the capacitor voltage of the CFC, $v_{o}(t)$ is the output voltage of CFC, $d(t)$ is the PWM duty cycle.

As for the 'buck mode', an average switching model of the CFC for the 'boost mode' can be derived based on equations (10) and (11) by calculating the average values of variables within one duty cycle:

$$
\left\{\begin{array}{l}
C \frac{d\left\langle v_{c}(t)\right\rangle_{T}}{d t}=\left(i_{s}(t)-i_{a}(t)\right) d(t)+i_{s}(t)(1-d(t))=i_{s}(t)-i_{a}(t) d(t) \\
\left\langle v_{o}(t)\right\rangle_{T}=v_{c}(t) d(t)
\end{array}\right.
$$

Substituting $v_{c}(t)=V_{c}+\hat{v}_{c}, \quad i_{s}(t)=I_{s}+\hat{i}_{s}, i_{a}(t)=I_{a}+\hat{i}_{a}, d(t)=D+\hat{d}$ into (12) and neglecting the second order and dc components, a linearized model can be obtained

$$
\left\{\begin{array}{l}
C \frac{d \hat{v}_{c}}{d t}=-\hat{i_{a}} D-\hat{d} I_{a}+\hat{i}_{s} \\
v_{o}=V_{c} \hat{d}+D \hat{v_{c}}
\end{array}\right.
$$

Fig. 5(b) gives the equivalent circuit of the small-signal model of the CFC in the 'boost mode'. By applying the Laplace transform to equation (13), a transfer function from the line current $\hat{i}_{a}$ to the duty cycle $\hat{d}$ is derived as follows:

$$
G_{i d}=\frac{\hat{i_{a}}}{\hat{d}}=-\frac{D I_{a}-V_{c} C s}{L C s^{2}+R C s+D^{2}}
$$

It should be emphasized that for the 'boost mode' representation a right half-plane (RHP) zero appears in the transfer function, as shown in equation (14). This indicates that the CFC under this operating mode will behave as a non-minimum phase system. The RHP zero causes an extra phase lag ( 90 degrees) in the frequency response of the system and, therefore, care should be exercised when designing a controller due to its implication on system stability. 


\section{Simulation results}

\section{A. System parameters}

An asymmetrical HVDC system (see Fig. 1) has been built in PSIM and PSCAD for the frequency analysis and time-domian simulaitons. The parameters of the system are listed in Table I. A switching frequency of $1 \mathrm{kHz}$ has been adopted in this paper to verify the operation of the CFC models. In a real project, the swiching frequency of the device would be selected based on the capability of the semiconductor devices. In an HVDC system, this value will be normally less than $1 \mathrm{kHz}$.

Table I: System parameters

\begin{tabular}{l|l}
\hline Parameter & Value \\
\hline Rated DC voltage & $500 \mathrm{kV}$ \\
\hline Rated power of VSC1 & $500 \mathrm{kVA}$ \\
\hline Rated power of VSC2 & $150 \mathrm{kVA}$ \\
\hline Rated power of VSC3 & $700 \mathrm{kVA}$ \\
\hline Inductor/ Resistor of Line a & $180 \mathrm{mH} / 2 \Omega$ \\
\hline Inductor/ Resistor of Line b & $150 \mathrm{mH} / 1 \Omega$ \\
\hline Inductor/ Resistor of Line c & $100 \mathrm{mH} / 0.2 \Omega$ \\
\hline Capacitor of the CFC & $20 \mathrm{mH}$ \\
\hline Switching of CFC & $1 \mathrm{kHz}$ \\
\hline Rated voltage of CFC & $8 \mathrm{kV}$ \\
\hline
\end{tabular}

\section{B. Frequency (s-domain) analysis in PSIM (AC Sweep)}

The meshed MTDC system upgraded with a dual H-bridge CFC, as shown in Fig. 3, has been built in PSIM to verify the validity and accuracy of the small-signal models presented in the previous section. An ac sweep procedure is performed, with small variations $\hat{d}$ being injected to the duty cycle $D$ (i.e. 0.05 p.u. from $0.1 \mathrm{~Hz}$ to $1 \mathrm{kHz}$ ). The current variations $\hat{i}_{a}$ in the transmission line are fed back to the ac sweep block (see Fig. 4). By analyzing signals $\hat{d}$ and $\hat{i}_{a}$, the frequency response of the system is obtained.
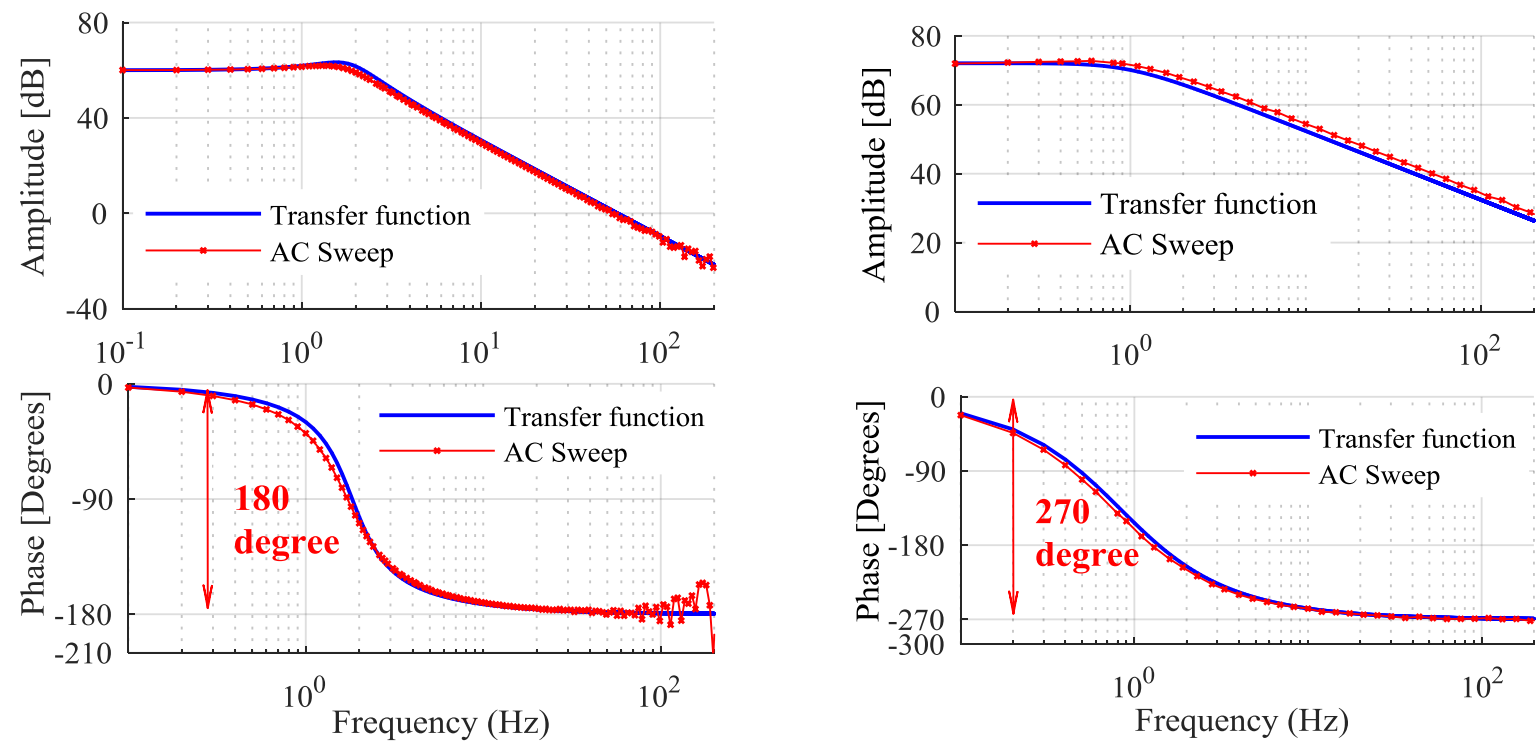

Fig. 6: Frequency response of the CFC (operating a duty cycle $D=0.5$ ). (a) 'Buck mode'. (b) 'Boost mode'. 
The frequency responses of the CFC in 'buck mode' and 'boost mode' are illustrated in Fig. 6(a) and Fig. 6(b), respectively. The blue curves are obtained in MATLAB using the transfer functions given by equations (9) and (14). The red curves are the results obtained through the ac sweep procedure in PSIM. By comparing these results, it can be seen that they match well. Quite notably, the phase lag intrinsic to the 'boost mode' operation is also exhibited when the ac sweep is performed, which is consistent with the analysis of the previous section.

\section{Time-domain simulations in PSCAD}

The three-terminal MTDC system shown in Fig. 1 has been built in PSCAD to assess the performance of the CFC. The time-domain simulation results are given in Fig. 7. The node current is regulated by VSC1 to $I_{1}=1 \mathrm{kA}$. It can be observed that the CFC operates in 'buck mode' (see area B) when currents $I_{a}$ and $I_{b}$ are smaller than the node current $I_{1}$. Conversely, the CFC operates in 'boost mode' when current $I_{a}$ (see area A) or $I_{b}$ (see area $\mathrm{C}$ ) is higher than the node current $I_{l}$ and the other current is reversed. These simulation results verify the analysis in previous sections.

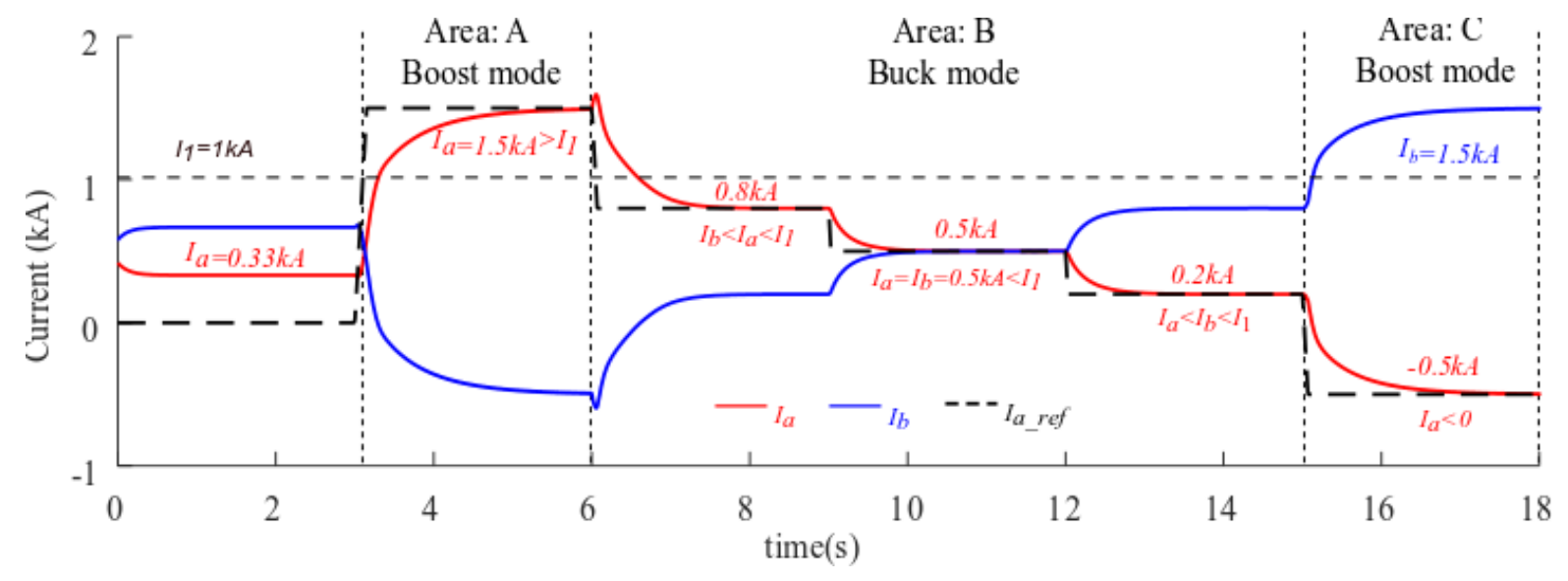

Fig. 7: Time-domain simulation results.

\section{Conclusion}

The modeling and operation of a dual H-bridge CFC have been analyzed in this paper. Following a topology simplification, the operating modes of the device have been classified into 'buck mode' and 'boost mode' based on the relationship between the node current and transmission line currents. Smallsignal models of the CFC have been derived for both operating modes. Due to their simplicity, they are suitable for control system design. It is shown that these models are structurally different for each mode of operation. Compared to the 'buck mode', the CFC working in 'boost mode' contains a right halfplane (RHP) zero and, hence, will exhibit a non-minimum phase system behavior. This leads to an extra 90 degrees phase lag in the frequency response of the system, which should be taken into consideration when designing the controller (i.e. the bandwidth of the system should be restricted to avoid instability).

A novel modeling approach was followed in this paper. The presented models enable a good understanding of the CFC performance based on its operating conditions. The validity and accuracy of the proposed representations have been verified through a frequency analysis in PSIM. The performance of the device has also been assessed via time-domain simulations conducted in PSCAD. The results support the definition of 'buck' and 'boost' operating modes of a dual H-bridge CFC.

\section{References}

[1] G. Li, C. Li, D. Van Hertem, "HVDC technology overview," in HVDC Grids: For Offshore and Supergrid of the Future, Hoboken, NJ, USA:Wiley, pp. 45-76, 2016. 
[2] D. Jovcic and K. Ahmed, High Voltage Direct Current Transmission: Converters, Systems, and DC Grids, University of Aberdeen, UK, Wiley, 2015.

[3] O. Cwikowski, J. Sau-Bassols, B. Chang, E. Prieto-Araujo, M. Barnes, O. Gomis-Bellmunt and R. Shuttleworth, "Integrated HVDC Circuit Breakers with Current Flow Control Capability," IEEE Trans. Power Del. vol.33, issue 1, pp. 371-380, Feb. 2018.

[4] E. Veilleux and B. Ooi, "Power flow analysis in multi-terminal HVDC grid," in Power Systems Conf. and Expo. (PSCE), pp. 1-7, 2011.

[5] D. Jovcic, M. Hajian, H. Zhang and G. Asplund, "Power flow control in dc transmission grids using mechanical and semiconductor-based dc/dc devices," in Proc. IET Int. Conf. AC DC Power Trans., 2012, pp. 1-6.

[6] E. Veilleux and B. T. Ooi, "Multiterminal HVDC With Thyristor Power-Flow Controller," IEEE Trans. Pow. Del., vol. 27, no. 3, pp. 1205-1212, 2012.

[7] C. Barker and R. Whitehouse, "A current flow controller for use in HVDC grids," in IET Int. Conf. on AC and DC Power Trans. (ACDC), pp. 1-5, 2012.

[8] F. Hassan, R. King, R. Whitehouse, and C. Barker, "Double modulation control (DMC) for dual H-bridge current flow controller (2B-CFC)," in 17th European Conf. on Power Electron. and Applications (EPE'15 ECCEEurope), Geneva, pp. 1-9, 2015.

[9] N. Deng, P. Wang, X. Zhang, G. Tang, and J. Cao, “A DC current flow controller for meshed modular multilevel converter multi-terminal HVDC grids," CSEE J. Power Energy Syst., vol. 1, no. 1, pp. 43-51, Mar. 2015.

[10] S. Balasubramaniam, C. E. Ugalde-Loo, J. Liang, T. Joseph, R. King and A. Adamczyk, "Experimental Validation of Dual H-Bridge Current Flow Controllers for Meshed HVDC Grids," IEEE Trans. Power Del. vol. 33, no. 1, pp. 381-392, Sep. 2017.

[11] J. Sau-Bassols, E. Prieto-Araujo and O. Gomis-Bellmunt, "Modelling and control of an interline Current Flow Controller for meshed HVDC grids," IEEE Trans. Power Del. vol. 32, issue 1, pp. 11-22, Feb. 2017.

[12] R. D. Middlebrook and S. Cuk, "A general unified approach to modeling switching-converter power stages," Int. J. Electronics, vol. 42, no.6, pp.521-550, June 1977. 